\title{
Language outcome related to brain structures in school-aged preterm children: A systematic review
}

\author{
Lottie W. Stipdonk $^{1 *}$, Marie-Christine J. P. Franken ${ }^{1}$, Jeroen Dudink ${ }^{2,3}$ \\ 1 Department of Otorhinolaryngology at Erasmus Medical University Centre-Sophia Children's Hospital, \\ Rotterdam, Netherlands, 2 Division of Neonatology, Department of Pediatrics at Erasmus Medical University \\ Centre-Sophia Children's Hospital, Rotterdam, Netherlands, 3 Division of Neonatology, Department of \\ Pediatrics at UMCU-Wilhelmina Children's Hospital, Utrecht, Netherlands \\ *I.stipdonk@erasmusmc.nl
}

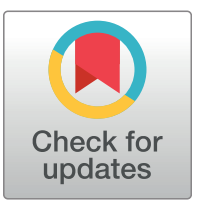

\section{openaccess}

Citation: Stipdonk LW, Franken M-CJP, Dudink J (2018) Language outcome related to brain structures in school-aged preterm children: A systematic review. PLoS ONE 13(6): e0196607. https://doi.org/10.1371/journal.pone.0196607

Editor: Nehal A. Parikh, Cincinnati Children's Hospital Medical Center, UNITED STATES

Received: January 9, 2018

Accepted: April 16, 2018

Published: June 4, 2018

Copyright: ๑ 2018 Stipdonk et al. This is an open access article distributed under the terms of the Creative Commons Attribution License, which permits unrestricted use, distribution, and reproduction in any medium, provided the original author and source are credited.

Data Availability Statement: All relevant data are within the paper and its Supporting Information files.

Funding: The authors received no specific funding for this work.

Competing interests: The authors have declared that no competing interests exist.

\section{Abstract}

Preterm children often have language problems. This atypical language development is probably due to atypical brain development. We conducted a systematic review to provide an overview of the extensive and diverse scientific literature on the relations between language outcome and underlying brain structures in school-aged preterm-born children. Embase, Medline Ovid, Web of Science, Cochrane central and Google scholar were searched for relevant studies. Inclusion criteria were: cases are school-aged preterm children; structural MRI (T1- and T2-weighted sequences) or DTI used in combination with a neurocognitive language test; publication in an English-language peer-reviewed journal. Correlational measures between language scores and brain volume or fractional anisotropy of a brain structure were extracted. 23 studies were included. The relations between oral language, verbal fluency and/or written language and MRI/DTI measurements of white matter, gray matter, cerebellum, corpus callosum and/or the fasciculi are presented. Oral language skills and verbal fluency appear to be related to the corpus callosum. Oral language skills are also related to the uncinate fasciculus. There seems to be no clear relation between cerebellar development and verbal fluency skills. Not one single brain area is responsible for atypical language development, but several brain areas and their connections are essential. For future research it is recommended to relate brain areas to oral language skills on a microstructural level in preterm children. We also recommend to use language tests in which it is possible to distinguish between several language domains, such as perceptive and expressive language.

\section{Introduction}

Technological advances and combined efforts of obstetricians and neonatologists have resulted in improved survival for preterm infants [1]. Nowadays, very preterm children ( $<32$ weeks) represent $1 \%-2 \%$ of all live births in developed countries [2]. These children are at risk for neurocognitive deficits even later in life. Depending on gestational age and neonatal complications, 
up to $30 \%$ of very preterm survivors in developed countries will experience significant longterm neurodevelopmental problems, such as cognitive, motor or hearing impairment [3]. Subtler neurodevelopmental impairments, such as language disorders, learning disabilities, attention deficits, behavioral problems and social-emotional difficulties, occur even more often [39]. Almost $20 \%$ of very preterm children are diagnosed with language disability at school age (6 to 17 years) and more than $50 \%$ require additional education [10]. Two recent meta-analyses showed that problems with complex language functions, such as storytelling, even increase at ages 3 to 13 years $[6,11]$. These outcomes are alarming since language development is extremely important for academic achievements and communication in everyday life.

The atypical language development in preterm children is most likely a consequence of atypical brain development [10, 12]. Several magnetic resonance imaging (MRI) studies showed macrostructural (e.g. measurements from T1- and T2 weighted structural MRI sequences) and microstructural (e.g. diffusion weighted MRI sequences) deviations in brain development in preterm children in childhood and adolescence [12]. As compared to termborn controls, very preterm children had significantly smaller total brain volume, white matter volume, gray matter volume, cerebellum, hippocampus and corpus callosum. Furthermore, preterm birth is associated with a reduction in cortical folding. In a recently published systematic review of the association between very low birth weight (VLBW) and brain structures and cognitive function impairments, the authors concluded that both brain structures and cognitive functions are more often atypically developed in VLBW children [13]. However, they did not look for association measurements between these two parameters and they did not include language outcome. Therefore, the association between atypical brain development and language skills remains unclear. Recently Kwon et al. reviewed literature about association measures between functional connectivity and language disorders [10]. The authors suggest that there are alterations in the functional organization of language in preterm children and that these alterations in the developing brain are both proximate and long lasting. However, to our knowledge no systematic review has been published about association measures between structural MRI measures and language development in preterm children, whilst many studies have addressed relations between different MRI brain structures and several language domains. These MRI studies are diverse, however, since they focus on the relation between two measurements (language outcomes and brain structure measurements), which both can vary. A clear overview of all these results could be of great use to clinicians and researchers in the field. It can contribute to a better understanding of the actual relations between language and the brain in preterm children and set directions for consistent and high-quality research. Hence, the aim of the systematic review presented here is to provide an overview of what is currently known about language outcome of school-aged preterm children and the associations with their brain structures measured on MRI.

\section{Method}

\section{Selection of studies}

The computerized Embase, Medline Ovid, Web of Science, Cochrane central and Google scholar databases were searched for articles in January 2017 (and again in September 2017 to detect recently published articles) combining the search terms neurological, neurophysiology, neurobiology, forebrain, brain AND speech, language, verbal, linguistic, reading, writing, literacy, illiteracy, vocabulary, grammar, phonology, dyslexia AND premature, prematurity, preterm, "low birth, birthweight", "small "gestational age". In Fig 1 the flow diagram of the study selection is presented. The search yielded 2083 unique articles. In S1 Text the complete search protocol can be found for all databases. Based on screening of titles and abstracts, 2035 articles were 


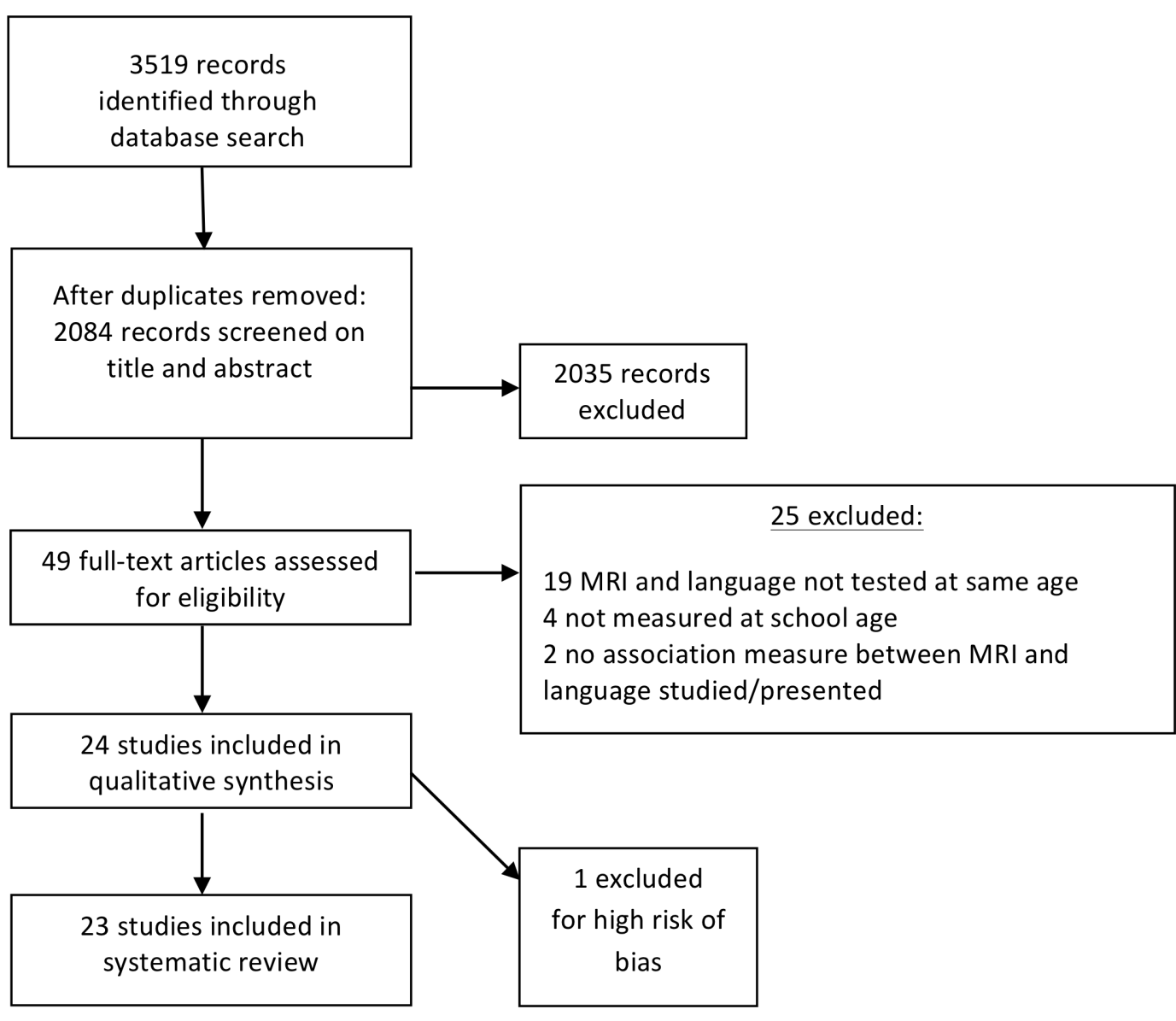

Fig 1. Flow diagram of study selection.

https://doi.org/10.1371/journal.pone.0196607.g001

excluded. 49 articles remained and were assessed for eligibility based on the following exclusion criteria: (1) study cases are not school-aged children (6-17 years) born preterm (gestational age (GA) <37 weeks); (2) brain structures not measured with structural MRI (T1- and T2-weighted scans) and/or DTI; (3) language not assessed at the same age as the MRI scan was made; (4) no correlational measure is published between language and brain volume or fractional anisotropy of a brain structure; (5) not published in an English language, peer-reviewed journal; (6) no sufficient study quality according to the Newcastle-Ottawa Quality Assessment Scale for cohort studies. A total of 25 studies had to be excluded based on these criteria, which resulted in 24 studies that were suitable for our data extraction and analysis (23 originally in January 2017, and 1 added in September 2017). Subsequently, one study was excluded since there was high risk of bias [14]; the population and main outcome measure of this study were overlapping with those of one of the other included studies [15]. Of these two studies, we included the most recently published one [15]. The main characteristics of the final 23 included studies are presented in Table 1.

\section{Quality assessment}

Two authors (LWS and JD) independently assessed the methodological quality of the included studies according to the Newcastle-Ottawa Quality Assessment Scale for cohort studies (S1 Table). This scale assesses the quality of cohort studies from the selection of the population, the comparability of the study groups and the ascertainment of outcome of interest. The total 
Table 1. Study characteristics.

\begin{tabular}{|c|c|c|c|c|c|c|c|c|}
\hline $\begin{array}{l}\text { Gäddlin et al. } \\
\text { [16] } 2008\end{array}$ & $\begin{array}{l}\text { PT: } \\
\text { 59FT: } 57\end{array}$ & $\begin{array}{l}<30 \\
\text { weeks }\end{array}$ & Down syndrome & 15 & $\begin{array}{l}\text { T1w, T2w } \\
\text { (6 different } \\
\text { hospitals) }\end{array}$ & $\begin{array}{l}\text { WM injury4-point } \\
\text { scale }\end{array}$ & $\begin{array}{l}\text { Oral language } \\
\text { (WISC)Written } \\
\text { language: } \\
\text { reading }\end{array}$ & 0 \\
\hline $\begin{array}{l}\text { Yliherva et al. } \\
\text { [17] } 2001\end{array}$ & $\begin{array}{l}\text { PT: } \\
\text { 41FT: } 24\end{array}$ & $\begin{array}{l}26-35 \\
\text { weeks }\end{array}$ & $\begin{array}{l}\text { Rett syndrome Duchenne } \\
\text { dystrophy }\end{array}$ & 8 & $1,0 \mathrm{~T} 1 \mathrm{w}$ & $\begin{array}{l}\text { WM injury4-point } \\
\text { scale }\end{array}$ & $\begin{array}{l}\text { Oral language } \\
\text { (ITPA, MT, } \\
\text { TTC) }\end{array}$ & 0 \\
\hline $\begin{array}{l}\text { Rushe et al. } \\
\text { [18] } 2001\end{array}$ & $\begin{array}{l}\text { PT: } \\
\text { 75FT: } 21\end{array}$ & $\begin{array}{l}<33 \\
\text { weeks }\end{array}$ & NA & $\begin{array}{l}14- \\
15\end{array}$ & $\begin{array}{l}\text { GE } 1,5 \\
\text { T1w, T2w }\end{array}$ & Injury3-point scale & $\begin{array}{l}\text { Verbal Fluency } \\
\text { (FAS, Object and } \\
\text { Animal naming, } \\
\text { BNT) Written } \\
\text { language } \\
\text { (SGRST) }\end{array}$ & 0 \\
\hline $\begin{array}{l}\text { Skranes et al. } \\
\text { [19] } 1997\end{array}$ & $\begin{array}{l}\text { PT: } \\
\text { 18FT: } 0\end{array}$ & $\begin{array}{l}<1500 \\
\text { gram }\end{array}$ & Disabled children (such as CP) & 6 & $\begin{array}{l}\text { Philips 1,5 } \\
\text { T1w, T2w }\end{array}$ & $\begin{array}{l}\text { Presence of } \\
\text { periventricular gliosis, } \\
\text { loss of white matter, } \\
\text { ventricular dilatation } \\
\text { and cortical atrophy }\end{array}$ & $\begin{array}{l}\text { Oral language } \\
\text { (WPSSI) }\end{array}$ & 0 \\
\hline $\begin{array}{l}\text { Isaacs et al. } \\
\text { [20] } 2004\end{array}$ & $\begin{array}{l}\text { PT: } \\
\text { 65FT: } 0\end{array}$ & $\begin{array}{l}28,5 \\
(1.2)\end{array}$ & $\begin{array}{l}\text { Neuromotor or neurosensory } \\
\text { impairment }\end{array}$ & $\begin{array}{l}12- \\
16\end{array}$ & $\begin{array}{l}\text { Siemens } \\
1,5 \mathrm{~T} 2 \mathrm{w}\end{array}$ & Brain volumes & $\begin{array}{l}\text { Oral language } \\
\text { (WISC) }\end{array}$ & $\begin{array}{l}\text { WM: +par/temp, }-\mathrm{fr}(V B M \\
\text { correlations: } 39,-69,28 ; p< \\
.01) \mathrm{GM}:-\mathrm{par},+\mathrm{fr}(V B M \\
\text { correlations: } \pm 40,-70,30 ; \\
\mathrm{p}<0.01)\end{array}$ \\
\hline \multirow[t]{2}{*}{$\begin{array}{l}\text { Nosarti et al. } \\
\text { [21] } 2008\end{array}$} & \multirow[t]{2}{*}{$\begin{array}{l}\text { PT: } \\
\text { 207FT: } \\
104\end{array}$} & \multirow[t]{2}{*}{$\begin{array}{l}<33 \\
\text { weeks }\end{array}$} & \multirow{2}{*}{$\begin{array}{l}\text { For controls: any history of } \\
\text { neurological conditions } \\
\text { including meningitis, head } \\
\text { injury and cerebral infections }\end{array}$} & \multirow[t]{2}{*}{$\begin{array}{l}14- \\
15\end{array}$} & \multirow[t]{2}{*}{ GE $1,5 \mathrm{~T} 1 \mathrm{w}$} & \multirow[t]{2}{*}{ Brain volumes } & $\begin{array}{l}\text { Verbal Fluency } \\
\text { (FAS, Object and } \\
\text { Animal naming) }\end{array}$ & $\begin{array}{l}\text { WM: }+ \text { fr/tempGM: }-\mathrm{fr} / \mathrm{temp} \\
(29 \% \text { of variance: } F=2,3 ; \\
p<0.0001)\end{array}$ \\
\hline & & & & & & & $\begin{array}{l}\text { Written language } \\
\text { (SGRST) }\end{array}$ & $\begin{array}{l}\mathrm{WM}:+\mathrm{fr} / \text { temp,GM:-fr/temp } \\
(28 \% \text { of variance } F=2,3 ; \\
p<0.0001)\end{array}$ \\
\hline $\begin{array}{l}\text { McCoy et al. } \\
\text { [22] } 2014\end{array}$ & $\begin{array}{l}\text { PT: } \\
\text { 26FT: } 0\end{array}$ & $\begin{array}{l}27,81 \\
(2.0)\end{array}$ & $\begin{array}{l}\text { Inclusion: liberal transfusion } \\
\text { group }\end{array}$ & 13 & $\begin{array}{l}\text { Siemens } \\
3,0 \mathrm{~T} 1 \mathrm{w}, \\
\mathrm{T} 2 \mathrm{w}\end{array}$ & Brain volumes & $\begin{array}{l}\text { Verbal Fluency } \\
\text { (COWAT) }\end{array}$ & $\begin{array}{l}\text { WM females: }+\operatorname{temp}\left(r^{2} \Delta=\right. \\
.237 ; p<.05) \text { Cerebellum: } 0\end{array}$ \\
\hline \multirow[t]{2}{*}{$\begin{array}{l}\text { Scott et al. } \\
\text { [23] } 2011\end{array}$} & \multirow[t]{2}{*}{$\begin{array}{l}\text { PT: } \\
218 \mathrm{FT}: \\
127\end{array}$} & \multirow[t]{2}{*}{$\begin{array}{l}<33 \\
\text { weeks }\end{array}$} & \multirow[t]{2}{*}{ NA } & \multirow[t]{2}{*}{$\begin{array}{l}14- \\
15\end{array}$} & \multirow[t]{2}{*}{$\begin{array}{l}\text { GE } \\
1,5 \mathrm{~T} 1 \mathrm{w} \\
\mathrm{T} 2 \mathrm{w}\end{array}$} & \multirow[t]{2}{*}{ Brain volumes } & $\begin{array}{l}\text { Verbal Fluency } \\
\text { (COWAT, } \\
\text { Object and } \\
\text { Animal Naming) }\end{array}$ & WM: 0fr \\
\hline & & & & & & & $\begin{array}{l}\text { Written language } \\
\text { (SGRST) }\end{array}$ & $\begin{array}{l}\text { WM: 0frGM: }+ \text { fr }(z \text {-score } \\
4.98 ; p<.05)\end{array}$ \\
\hline $\begin{array}{l}\text { Arhan et al. } \\
\text { [24] } 2017\end{array}$ & $\begin{array}{l}\text { PT: } \\
\text { 22FT: } 24\end{array}$ & $\begin{array}{l}28-33 \\
\text { weeks }\end{array}$ & $\begin{array}{l}\text { Apgar score at } 5 \mathrm{~min}>7 ; \\
\text { absence of major neonatal } \\
\text { morbidity; absence of cerebral } \\
\text { pathology such as IVH }\end{array}$ & 9 & $\begin{array}{l}\text { Siemens } \\
1,5 \mathrm{~T} 1 \mathrm{w}\end{array}$ & $\begin{array}{l}\text { Cerebellum and CC } \\
\text { volume }\end{array}$ & $\begin{array}{l}\text { Oral language } \\
\text { (WISC) }\end{array}$ & $\begin{array}{l}\text { Cerebellum: }+(\text { Subtest } \\
\text { comprehension: } r=.93 ; p= \\
.001) \text { CC: }+(\text { subtest } \\
\text { vocabulary: } r=.91 ; p=.001)\end{array}$ \\
\hline \multirow[t]{2}{*}{$\begin{array}{l}\text { Parker et al. } \\
\text { [25] } 2008\end{array}$} & \multirow[t]{2}{*}{$\begin{array}{l}\text { PT: } \\
\text { 65FT: } 34\end{array}$} & \multirow[t]{2}{*}{$\begin{array}{l}<33 \\
\text { weeks }\end{array}$} & \multirow[t]{2}{*}{$\begin{array}{l}\text { IVH, drug exposure during } \\
\text { pregnancy }\end{array}$} & \multirow[t]{2}{*}{15} & \multirow[t]{2}{*}{ GE $1,5 \mathrm{~T} 1 \mathrm{w}$} & \multirow[t]{2}{*}{ Cerebellum volume } & $\begin{array}{l}\text { Oral language } \\
\text { (WISC) }\end{array}$ & $+(r=.401 ; p=.008)$ \\
\hline & & & & & & & $\begin{array}{l}\text { Verbal Fluency } \\
\text { (COWAT, } \\
\text { Object and } \\
\text { Animal Naming) }\end{array}$ & 0 \\
\hline \multirow[t]{2}{*}{$\begin{array}{l}\text { Narberhaus } \\
\text { et al.[26] } 2008\end{array}$} & \multirow[t]{2}{*}{$\begin{array}{l}\text { PT: } \\
\text { 52FT: } 52\end{array}$} & \multirow[t]{2}{*}{$\begin{array}{l}<33 \\
\text { weeks }\end{array}$} & \multirow{2}{*}{$\begin{array}{l}\text { IQ }<70 \text {, history of traumatic } \\
\text { brain injury, CP or other } \\
\text { neurological diagnosis, motor or } \\
\text { sensory impairment that } \\
\text { precluded neuropsychological } \\
\text { assessment }\end{array}$} & \multirow[t]{2}{*}{$\begin{array}{l}10- \\
19\end{array}$} & \multirow[t]{2}{*}{ GE $1,5 \mathrm{~T} 1 \mathrm{w}$} & \multirow[t]{2}{*}{ CC volume } & $\begin{array}{l}\text { Oral language } \\
\text { (WISC/WAIS } \\
\text { subtest) }\end{array}$ & $\begin{array}{l}+(\text { for splenium: } r=.32 ; p< \\
.05)\end{array}$ \\
\hline & & & & & & & $\begin{array}{l}\text { Verbal fluency } \\
\text { (COWAT) }\end{array}$ & $\begin{array}{l}+(\text { for genu: } r=.37 ; p<.01) \\
\text { (for splenium: } r=0.32 ; p< \\
.05) \text { (for isthmus: } r=.28 ; p< \\
.05)\end{array}$ \\
\hline
\end{tabular}


Table 1. (Continued)

\begin{tabular}{|c|c|c|c|c|c|c|c|c|}
\hline \multirow[t]{3}{*}{$\begin{array}{l}\text { Nosarti et al. } \\
\text { [27] } 2004\end{array}$} & \multirow[t]{3}{*}{$\begin{array}{l}\text { PT: } \\
\text { 66FT: } 51\end{array}$} & \multirow[t]{3}{*}{$\begin{array}{l}<33 \\
\text { weeks }\end{array}$} & \multirow[t]{3}{*}{ NA } & \multirow[t]{3}{*}{$\begin{array}{l}14- \\
15\end{array}$} & \multirow{3}{*}{$\begin{array}{l}\text { GE } \\
1,5 \mathrm{~T} 1 \mathrm{w} \\
\mathrm{T} 2 \mathrm{w}\end{array}$} & \multirow[t]{3}{*}{ CC volume } & $\begin{array}{l}\text { Oral language } \\
\text { (WISC) }\end{array}$ & $\begin{array}{l}+(\text { for mid-posterior CC: } \beta= \\
.33 ; p=.009)\end{array}$ \\
\hline & & & & & & & $\begin{array}{l}\text { Verbal fluency } \\
\text { (COWAT, } \\
\text { Object and } \\
\text { Animal Naming) }\end{array}$ & $+($ total $C C: \beta=.35 ; p=.006)$ \\
\hline & & & & & & & $\begin{array}{l}\text { Written language } \\
\text { (SGRST) }\end{array}$ & 0 \\
\hline \multirow[t]{2}{*}{$\begin{array}{l}\text { Allin et al. } \\
{[28] 20^{\prime} 01}\end{array}$} & \multirow[t]{2}{*}{$\begin{array}{l}\text { PT: } \\
\text { 67FT: } 50\end{array}$} & \multirow[t]{2}{*}{$\begin{array}{l}<33 \\
\text { weeks }\end{array}$} & \multirow[t]{2}{*}{ NA } & \multirow[t]{2}{*}{$\begin{array}{l}14- \\
15\end{array}$} & \multirow[t]{2}{*}{ GE $1,5 \mathrm{~T} 1 \mathrm{w}$} & \multirow[t]{2}{*}{ Cerebellum volume } & $\begin{array}{l}\text { Verbal Fluency } \\
\text { (FAS, BNT) }\end{array}$ & 0 \\
\hline & & & & & & & $\begin{array}{l}\text { Written language } \\
\text { (SGRST) }\end{array}$ & $\begin{array}{l}\text { Reading: }+(\beta=.295 ; p= \\
.019) \text { Spelling: } 0\end{array}$ \\
\hline $\begin{array}{l}\text { Martinussen } \\
\text { et al.[29] } 2009\end{array}$ & $\begin{array}{l}\text { PT: } \\
\text { 50FT: } \\
\text { 49SGA: } \\
49 \\
\end{array}$ & $\begin{array}{l}29,1 \\
(2.7)\end{array}$ & NA & 15 & $\begin{array}{l}\text { Siemens } \\
1,5 \mathrm{IR}\end{array}$ & Brain volumes & $\begin{array}{l}\text { Oral language } \\
\text { (WISC) }\end{array}$ & $\begin{array}{l}\text { 0WM and Cerebellum: } \\
+ \text { SGA(Stepwise regression: } \\
\text { step } 2, \text { part } r=.1066, F \\
\text { value }=6.53 ; p=.0142)\end{array}$ \\
\hline \multirow[t]{3}{*}{$\begin{array}{l}\text { Brumbaugh } \\
\text { et al.[30] } 2016\end{array}$} & \multirow[t]{3}{*}{$\begin{array}{l}\text { PT: } \\
\text { 52FT: } 74\end{array}$} & \multirow[t]{3}{*}{$\begin{array}{l}34-36 \\
\text { weeks }\end{array}$} & \multirow{3}{*}{$\begin{array}{l}\text { Multiple birth, major medical } \\
\text { disease, neurological injury, } \\
5 \text {-minute Apgar score }<7 \text {, } \\
\text { neonatal sepsis, and birth weight } \\
<1500 \text { g for late PT children and } \\
<2500 \text { g for FT children }\end{array}$} & \multirow[t]{3}{*}{$\begin{array}{l}6- \\
13\end{array}$} & \multirow[t]{3}{*}{$\begin{array}{l}\text { Siemens } \\
\text { 3,0T1w }\end{array}$} & \multirow[t]{3}{*}{$\begin{array}{l}\text { Brain volumes (WM, } \\
\text { Cerebellum) }\end{array}$} & $\begin{array}{l}\text { Oral language } \\
\text { (WISC) }\end{array}$ & 0 \\
\hline & & & & & & & $\begin{array}{l}\text { Verbal Fluency } \\
\text { (BNT, Object } \\
\text { Naming) }\end{array}$ & 0 \\
\hline & & & & & & & $\begin{array}{l}\text { Written } \\
\text { language: } \\
\text { reading (WRAT) }\end{array}$ & 0 \\
\hline \multirow[t]{2}{*}{$\begin{array}{l}\text { Caldú et al. } \\
\text { [31] } 2006\end{array}$} & \multirow[t]{2}{*}{$\begin{array}{l}\text { PT: } \\
\text { 25FT: } 25\end{array}$} & \multirow{2}{*}{$\begin{array}{l}<33 \\
\text { weeks, } \\
29.48 \\
(2.52)\end{array}$} & \multirow[t]{2}{*}{$\begin{array}{l}\text { Mentally or physically disabled } \\
\text { children }\end{array}$} & \multirow[t]{2}{*}{13} & \multirow[t]{2}{*}{ GE $1,5 \mathrm{~T} 1 \mathrm{w}$} & \multirow[t]{2}{*}{$\begin{array}{l}\text { GM, WM and CC } \\
\text { volume }\end{array}$} & $\begin{array}{l}\text { Oral language } \\
\text { (WISC/WAIS), }\end{array}$ & $\begin{array}{l}\text { WM: 0GM: }+(r=.50 ; p< \\
.05) \text { CC: } 0\end{array}$ \\
\hline & & & & & & & $\begin{array}{l}\text { Verbal fluency } \\
\text { (RAVLT) }\end{array}$ & WM: 0GM: 0CC: 0 \\
\hline $\begin{array}{l}\text { Northam } \\
\text { et al.[32] } 2012\end{array}$ & $\begin{array}{l}\text { PT: } \\
\text { 50FT: } 30\end{array}$ & $\begin{array}{l}<33 \\
\text { weeks. } \\
27(2)\end{array}$ & NA & 16 & $\begin{array}{l}\text { Siemens } \\
1,5 \mathrm{~T} 1 \mathrm{w} \\
\mathrm{T} 2 \mathrm{w}\end{array}$ & $\begin{array}{l}\text { Brain volumes (CC, } \\
\text { Fasciculi) }\end{array}$ & $\begin{array}{l}\text { Oral language } \\
\text { (PPVT, TROG) }\end{array}$ & $\begin{array}{l}\text { CC: }+(\text { Ancova } F(2.72)=20.5 \\
p<.0001) \text { UF: }+ \text { AF: } 0\end{array}$ \\
\hline \multirow[t]{2}{*}{$\begin{array}{l}\text { Mullen et al. } \\
\text { [33] } 2011\end{array}$} & \multirow[t]{2}{*}{$\begin{array}{l}\text { PT: } \\
\text { 44FT: } 41\end{array}$} & \multirow[t]{2}{*}{$\begin{array}{l}28,3 \\
(1.9)\end{array}$} & \multirow{2}{*}{$\begin{array}{l}\text { IVH, PVL, low pressure } \\
\text { ventriculomegaly, abnormal } \\
\text { MRI findings, abnormal total } \\
\text { ventricular CSF volume }\end{array}$} & \multirow[t]{2}{*}{16} & \multirow[t]{2}{*}{$\begin{array}{l}\text { Siemens } \\
1,5\end{array}$} & \multirow[t]{2}{*}{ Brain volumes } & $\begin{array}{l}\text { Oral language } \\
\text { (WISC, PPVT) }\end{array}$ & 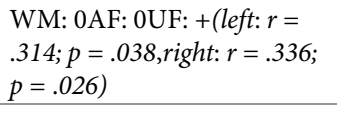 \\
\hline & & & & & & & $\begin{array}{l}\text { Verbal Fluency } \\
\text { (CTOPP) }\end{array}$ & $\begin{array}{l}\text { AF: }+(\text { left: } r=.424 ; p=.004 \\
\text { right: } r=.301 ; p=.047) \mathrm{UF}: 0\end{array}$ \\
\hline $\begin{array}{l}\text { Andrews et al. } \\
\text { [34] } 2010\end{array}$ & $\begin{array}{l}\text { PT: } \\
\text { 19FT: } 9\end{array}$ & $\begin{array}{l}24-36 \\
\text { weeks } \\
30,5 \\
(3,2)\end{array}$ & NA & 11 & $\begin{array}{l}\text { Siemens } \\
\text { 3,0T1w }\end{array}$ & DTI & $\begin{array}{l}\text { Written language } \\
\text { : reading (WJTA) }\end{array}$ & $\begin{array}{l}\text { Temp/par: }+(\text { for passage } \\
\text { comprehension: left: } r=.417 ; \\
p<.05, \text { right: } r=.459 ; p< \\
.05) \text { CC: }+(\text { for } \text { word } \\
\text { identification: } r=.553 ; p< \\
.05 \text { for word attack: } r=.537 ; p \\
<.05)\end{array}$ \\
\hline $\begin{array}{l}\text { Constable } \\
\text { et al.[35] } 2008\end{array}$ & $\begin{array}{l}\text { PT: } \\
\text { 29FT: } 22\end{array}$ & $\begin{array}{l}28,4 \\
(2,0)\end{array}$ & $\begin{array}{l}\text { IVH, WM injury and/or } \\
\text { ventriculomegaly }\end{array}$ & 12 & $\begin{array}{l}\text { GE } \\
1,5 \text { SPGR }\end{array}$ & DTI & $\begin{array}{l}\text { Oral language } \\
\text { (WISC, PPVT-R) }\end{array}$ & $\begin{array}{l}\text { UF: +males(for VIQ left: } \\
r=0.513 ; p=.051, \text { right }: r= \\
.635 ; p=.008 \text { for PPVT left: } r \\
=.511 ; p=.052, \text { right }: r= \\
.619, p=.011 \text { UFright: }- \\
\text { females(for VIQ: } r=-.744 ; p \\
=.004 \text { for PPVT: } r=-.759, p= \\
.003)\end{array}$ \\
\hline
\end{tabular}


Table 1. (Continued)

\begin{tabular}{|c|c|c|c|c|c|c|c|c|}
\hline \multirow[t]{2}{*}{$\begin{array}{l}\text { Kontis et al. } \\
\text { [36] } 2009\end{array}$} & \multirow[t]{2}{*}{$\begin{array}{l}\text { PT: } \\
\text { 63FT: } 45\end{array}$} & \multirow[t]{2}{*}{$\begin{array}{l}<33 \\
\text { weeks }\end{array}$} & \multirow[t]{2}{*}{ Left-handedness } & \multirow[t]{2}{*}{15} & \multirow[t]{2}{*}{ GE 1,5} & \multirow[t]{2}{*}{ DTI } & $\begin{array}{l}\text { Oral language } \\
\text { (WISC) }\end{array}$ & CC: 0 \\
\hline & & & & & & & $\begin{array}{l}\text { Verbal Fluency } \\
\text { (CVLT) }\end{array}$ & $\begin{array}{l}\text { CC: }+(\text { for CC body with } \\
\text { intrusions item: } r=.295 ; p= \\
.029 \text { for Splenium with short } \\
\text { delay }: r=.312, p=.020 \text { for } \\
\text { splenium with long delay: } r= \\
.273 p=.0 .44 \text { for splenium } \\
\text { with long delay free recall: } r= \\
.313, p=.020 \text { for splenium } \\
\text { with intrusions: } r=-.306, p= \\
.023)\end{array}$ \\
\hline $\begin{array}{l}\text { Skranes et al. } \\
\text { [37] } 2007\end{array}$ & $\begin{array}{l}\text { PT: } \\
\text { 34FT: } 47\end{array}$ & $\begin{array}{l}29,3 \\
(2.7)\end{array}$ & Trisomy 21 & 15 & $\begin{array}{l}\text { Siemens } \\
1,5 \mathrm{~T} 1 \mathrm{w}\end{array}$ & DTI & $\begin{array}{l}\text { Oral language } \\
\text { (WISC) }\end{array}$ & SLright: $+(r=.363 ; p<0.05)$ \\
\hline $\begin{array}{l}\text { Travis et al. } \\
\text { [15] } 2016\end{array}$ & $\begin{array}{l}\text { PT: } \\
\text { 26FT: } 19\end{array}$ & $\begin{array}{l}26,0- \\
34,5\end{array}$ & $\begin{array}{l}\text { Active seizure disorder, } \\
\text { hydrocephalus, receptive } \\
\text { vocabulary score }<70, \\
\text { sensorineural hearing loss, and } \\
\text { non-native speaker of English }\end{array}$ & $\begin{array}{l}9- \\
17\end{array}$ & GE 3,0T1w & DTI & $\begin{array}{l}\text { Written } \\
\text { language: } \\
\text { reading (WJTA; } \\
\text { BRSC) }\end{array}$ & $\begin{array}{l}\text { AFleft: }+ \text { (for decoding: } r= \\
.606 ; p<.05) \text { UFleft: }+(\text { for } \\
\text { comprehension: } r=.562 ; p< \\
.05) \text { SL: }+(\text { for decoding: } r i g h t: \\
r=.403 ; p<.05), \text { left }: r= \\
.466 ; p<.05 \text { for } \\
\text { comprehension: left: } r=.417 \\
p<.05)\end{array}$ \\
\hline
\end{tabular}

In the correlation column, a '+' refers to a positive correlation; a '-' refers to a negative correlation; a ' 0 ' refers to no significant correlation.

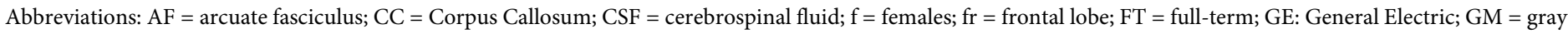

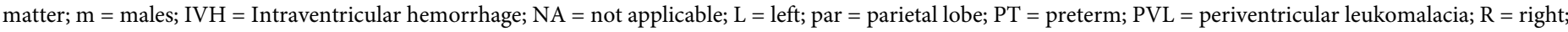

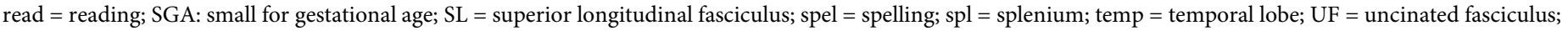
$\mathrm{WM}=$ white matter.

rating score ranges from 1 to 9 , with 9 being the most favorable. Any disagreement between the two assessors with regard to the total score was resolved by discussion. The quality scores of selected articles are presented in S1 Table. Overall quality was rated from 5 to 8 stars. To improve our reporting the PRISMA checklist was used (S2 Table).

\section{Outcome measures}

Language outcome. Language is a very complex phenomenon which encompasses many different subdomains. Most language tests represent only one of these subdomains, assessed by associated language tasks. Therefore, not all language studies can be compared in a single, consistent way. Only studies that used the same language task, or comparable ones measuring the same language domain, can be validly compared. For example, composing and speaking a complex sentence is a task that is completely different from summing up words that start with an F, or spelling individual words-each of these three tasks requires skills from a specific language domain. Inevitably, the language tests used in the included studies vary widely. To be able to still validly compare study results we created three categories: oral language; verbal fluency and written language.

The oral language category includes tests that assess oral language ability, such as word and sentence comprehension and production, and vocabulary. Included tests are: Clinical Evaluation of Language Fundamentals-4 (CELF); Illinois Test of Psycholinguistic Abilities (ITPA); Morphological Test (MT); Peabody Picture Vocabulary Test (PPVT); Test for Reception of Grammar (TROG); Token Test for Children (TTC); Verbal scale of Wechsler Intelligence Scale for Children-III (WISC); verbal scale of Wechsler Adult Intelligence Scale (WAIS); verbal scale of Wechsler Preschool and Primary Scale of Intelligence (WPSSI). 
The verbal fluency category includes tests that assess verbal (phonetic or semantic) fluency, which requires special use of executive functions in combination with language functions: Boston Naming Test (BNT); Controlled Oral Word Association Test (COWAT); Comprehensive Test of Phonological Processing (CTOPP); California Verbal Learning Test (CVLT); FAS-test; Object and Animal naming; Rey Auditory Verbal Learning Test (RAVLT); Stroop test TBAG version.

The written language category includes tests that target reading and spelling: Basic Reading Skills Cluster (BRSC); Schonnel Graded Reading and Spelling Test (SGRST); reading subtests of the Woodstock-Johnson III Test of Achievement (WJTA); reading score of Wide Range Achievement Test (WRAT).

MRI. We related language outcome in the above-mentioned three categories to the underlying brain structures. Different brain structures can be reliably measured in-vivo on structural (anatomical) T1- and T2-weighted MRI sequences-either manually, semi-automatically or automatically. Different software post processing tools are available for this purpose, allowing macro-structural measurements of brain structures. Diffusion tensor imaging (DTI) sequences allow visualization and quantification of white and gray matter microstructure. Several diffusion parameters can be derived from DTI results, but white matter integrity is most commonly estimated with fractional anisotropy (FA). FA is a scalar value between 0 and 1 describing the amount of diffusion asymmetry (anisotropy) within a voxel, defined in terms of its eigenvalues. $\mathrm{FA}=0$ means that diffusion is isotropic (i.e. it is unrestricted or equally restricted in all directions). FA $=1$ means that diffusion occurs along one axis only and is fully restricted along all perpendicular directions.

\section{Data extraction}

For each included study we extracted the published correlational measures between language scores and brain volume or fractional anisotropy of a brain structure. Most of the studies reported a Pearson's correlation coefficient. A few studies also reported Spearman's rho or stepwise logistic regression analyses as a correlation measure. The correlational measure had to correlate a language score (classified within one of the three categories discussed above) and a brain area that is addressed in at least two different studies. For example, total brain volume, brainstem volume and cerebral spinal fluid (CSF) were all studied only once [29-31], and therefore the respective findings are not presented in the cross table. Besides, these studies explicitly reported that they did not find any significant relations with language.

\section{Results}

Four of the 23 included studies [16-19] addressed the relation between children's language skills and white matter injury only, classified on either a 3- or 4-point scale. None of these studies found a significant association between this damage classification and language skills.

The remaining 19 studies used brain volume measurements or DTI to relate brain structures to language outcomes. A cross table (Table 2) shows the associations between language skills and brain structures reported in these 19 studies. A '+' refers to a positive correlation, a

'-' to a negative correlation and a ' 0 ' to no significant correlation.

\section{Oral language}

White and gray matter volume. Five studies reported findings about total white matter (WM) and/or gray matter (GM) volumes and the correlation with oral language scores [20, 29-31, 33]. Four studies [29-31, 33] explicitly reported no significant correlations with WM volume in preterm children. However, one of these [29] did find a significant correlation for 
Table 2. Study results.

\begin{tabular}{|c|c|c|c|c|c|c|}
\hline & & & & Arcuate & Uncinate & $\begin{array}{l}\text { Superior } \\
\text { Longitudinal }\end{array}$ \\
\hline $\begin{array}{l}+ \text { SGA }[29],+ \text { par/temp }[20],-f r \\
{[20]}\end{array}$ & $\begin{array}{l}+[31],+ \text { fr }[20]-\text { par } \\
{[20]}\end{array}$ & $\begin{array}{l}+\operatorname{spl}[26],+[27]^{\prime}[24, \\
32]\end{array}$ & $\begin{array}{l}+ \text { SGA[29], +[24, } \\
25]\end{array}$ & & $\begin{array}{l}+[32,33] \mathrm{L} / \mathrm{R},+\mathrm{m}[35],-\mathrm{f} \mathrm{R} \\
{[35]}\end{array}$ & $+\mathrm{R}[37]$ \\
\hline $0[29,31]^{\prime}[30]^{\prime}[33]$ & & $0[31,36]$ & $0[29]^{\prime}[30]$ & $\begin{array}{l}0[32,33] \mathrm{L} / \\
\mathrm{R}\end{array}$ & & \\
\hline +fr/temp[21] +temp_f[22] & -fr/temp[21] & $+[26,27,36]$ & & $+[33] \mathrm{L} / \mathrm{R}$ & & \\
\hline $0[30,31], 0 \mathrm{fr}[23]$ & $0[31]$ & $0[31]$ & $0[22,25,28,30]$ & & $0[33] \mathrm{L} / \mathrm{R}$ & \\
\hline +fr/temp[21] +temp/par[34] & $-\mathrm{fr} / \mathrm{temp}[21]+\mathrm{fr}[23]$ & $+[34]$ & $+\operatorname{read}[28]$ & $+\mathrm{L}[15]$ & $+\mathrm{L}[15]$ & $+\mathrm{L} / \mathrm{R}[15]$ \\
\hline $0[30], 0 f r[23]$ & & $0[27]$ & $0[30], 0$ spel[28] & & & \\
\hline
\end{tabular}

A ' + ' refers to a positive correlation; a '-' refers to a negative correlation; a ' 0 ' refers to no significant correlation.

Abbreviations: $\mathrm{CC}=$ Corpus Callosum; $\mathrm{f}=$ females; $\mathrm{fr}=$ frontal lobe; $\mathrm{GM}=$ gray matter; $\mathrm{m}=$ males; $\mathrm{L}=$ left; par = parietal lobe; $\mathrm{R}=$ right; read = reading; $\mathrm{SGA}$ : small for gestational age; spel = spelling; $\mathrm{spl}=$ splenium; temp = temporal lobe; $\mathrm{WM}=$ white matter .

small for gestational age (SGA) children. Also, two studies [20,31] found significant correlations in preterm children; the authors emphasized that these correlations are based on complicated, specific patterns of cortical and subcortical alterations. For example, Isaacs et al. described a positive correlation with WM volume in specific areas of the parietal and temporal lobes, a negative correlation between language and WM volume in frontal lobe areas, a negative correlation with GM volume in the parietal lobe and a positive correlation with GM volume in the frontal lobe [20]. Thus, both positive and negative correlations between oral language and GM and WM volume in different cortical areas were found.

Corpus callosum volume. Six studies described a relation between the volume of the corpus callosum (CC) and oral language skills. Four of them presented a positive correlation [24, $26,27,32$ ]. Arhan et al. [24] even show a correlation of $r=0.91 ; p=0.001$, which can be interpreted as very strong. Caldu et al. [31] and Kontis et al. [36] did not find a significant correlation between oral language skills and CC volume.

Cerebellum volume. Four studies associated cerebellar volume with oral language skills. Arhan et al. [24] and Parker et al. [25] described a positive correlation between oral language skills and cerebellar volume in preterm children. Arhan et al. again show a very strong association $(\mathrm{r}=0.93 ; \mathrm{p}=0.001)$. Two studies did not find a correlation in preterm children $[29,30]$. Martinussen et al. [29] did find a correlation in SGA children though.

DTI measurements fasciculi. Five studies reported findings about the association between oral language skills and the fasciculi in the brain. None of the studies reported a significant relation between oral language skills and the arcuate fasciculus (AF). Moreover, two studies reported explicitly that no significant relation was found between oral language skills and the AF [32, 33]. However, three studies reported a significant positive relation between oral language and the uncinate fasciculus (UF) $[32,33,35]$. The correlations presented by Constable et al. [35] are worth to note specifically, since the associations they found were strong (for example $\mathrm{r}=-0.759 ; \mathrm{p}=.003$ for the association between PPVT scores and the right UF in females). One study reported a significant positive relation with the superior longitudinal fasciculus (SLF) [37].

\section{Verbal fluency: Language and executive functioning}

White and gray matter volume. Five studies reported findings about the relation between WM and/or GM volume and verbal fluency skills. Two of these studies described a significant correlation [21, 22]. The one, by McCoy et al. [22], found a positive correlation in females, in 
higher temporal white matter. The other, by Nosarti et al. [21], found a positive correlation between WM volume in frontal and temporal regions and verbal fluency and a negative correlation between GM volume and verbal fluency. The other three studies did not find any correlation between verbal fluency and GM or WM volumes [23, 30, 31].

Corpus callosum volume. Five studies reported findings about the correlation between CC volume and verbal fluency. Three studies $[26,27,36]$ described a positive correlation. However, Caldu et al. [31] did not find any correlation between CC and verbal fluency.

Cerebellum volume. None of the four studies that described the relation between cerebellar volume and verbal fluency found any correlation $[22,25,28,30]$.

DTI measurements fasciculi. Only Mullen et al. [33] reported about the relation between fasciculi and verbal fluency and found a significant positive correlation with the left and right $\mathrm{AF}$ and no correlation with the UF.

\section{Written language: Reading and spelling}

White and gray matter volume. Four studies reported findings about GM and/or WM volume in relation with written language skills. Nosarti et al. [21] found significant correlations in the temporal gyrus: negative correlation with GM volumes and positive correlation with WM volumes in females only. Andrews et al. [34] also found a positive correlation in temporal parietal regions between reading and WM volume. Scott et al. [23] presented a positive correlation between GM volume in frontal lobe regions and no correlations, however, with WM volumes. Brumbaugh et al. [30] did not find significant correlations between WM volume and written language skills.

Corpus callosum volume. Andrews et al. [34] found a significant correlation between fractional anisotropy in the CC and reading skills. On the other hand, Nosarti et al. [27] did not find any significant correlations between CC volume and written language skills.

Cerebellum volume. Allin et al. [28] found a positive correlation between reading skills and cerebellar volume, but not between spelling skills and cerebellar volume. Brumbaugh et al. [30] did not find a correlation between reading and cerebellum volume.

DTI measurements fasciculi. Only one study, by Travis et al., described correlations between fractional anisotropy in the fasciculi and written language skills [15]. Correlations were found with reading and spelling and the left AF and left UF.

\section{Discussion}

\section{Main findings}

Our overview of study results in language and brain structure associations in preterm children yielded a complex set of relations, of which some show more consensus than others. We will discuss the most remarkable results.

Perhaps most notable is the lack of any association between structural brain injury and language outcomes. We had expected that preterm children with explicit brain damage would have the most severe language problems. However, in these studies brain damage was scored on a 3- or 4-point scale and naturally, in all studies the group of children with explicit damage was relatively small compared to groups of children with less damage, which makes it hard to prove a correlation with language. This might have influenced the correlations found between language skills and brain damage.

Another remarkable result concerns the cerebellum volume. We studied three language domains (i.e. oral language, verbal fluency and written language) and only very few studies found a significant correlation between any of these domains and the volume of the cerebellum; no correlation at all was reported for verbal fluency. A clear correlation between verbal fluency and cerebellar volume cannot be shown, and seems unlikely for both oral and written language. 
The association of the $\mathrm{CC}$ volume with language outcomes is more convincing, particularly with regard to oral language skills and verbal fluency. Only one of the included studies did not find a significant correlation with language skills or verbal fluency [31], but this can likely be ascribed to insufficient statistical power, as this was the study with the smallest population of preterm children $(\mathrm{N}=25)$. Overall, an association between oral language and $\mathrm{CC}$ volume is likely. However, the relation between CC and written language skills remain inconclusive, since there were only two studies that reported correlational data between these measures and they reported opposing results [27, 34].

Regarding the DTI studies, the most striking result is the repeatedly reported significant correlation between UF and oral language skills. The UF is part of the ventral language pathway and in recent literature it is often associated with semantic language functions. However, there is a lack of evidence for a general role of the UF in language [38]. Our review, though, showed a positive correlation between the UF and oral language skills. An association between language skills and the AF, which is part of the dorsal language stream, is less obvious according to our review results. Unfortunately, few studies included in our review addressed the role of the AF. Still, these tentatively show that the AF is more involved in verbal fluency, whilst the UF is more involved in oral language.

A less convincing result is the correlation between language and areas of WM and GM volume. Many studies did look at WM and GM in relation to one of the language domains, but the results were inconsistent. We propose that these kinds of differences between studies might arise because each addresses a slightly different microstructural area of the brain. When total WM or GM volume is studied, rarely any relation with language is found, while many significant relations are found when studying several microstructures of the brain. For example,

Nosarti et al. [21] found a negative correlation between written language skills and GM volume in the temporal lobe, while Scott et al. [23] found a positive correlation in the frontal lobe.

Overall we see that GM volume is more often negatively correlated with language skills, while WM volume mostly correlated positively with language skills. This negative association of GM with language corresponds with recent literature, also for example in stuttering literature [39], and has been associated with a cortical developmental phase of dendritic and synaptic pruning in late childhood and adolescence [40-43]. This might mark a shift from relatively diffuse cortical representations of cognitive functions in early childhood toward a more accurate, efficient, and faster processing language system later on.

Oral language skills are more often significantly correlated to preterm brain structures compared to verbal fluency skills or written language skills (see Table 2). Thus, atypical brain development in preterm children seems to affect oral language more obviously than it affects verbal fluency or reading or spelling. This is interesting in the sense that verbal fluency skills are also based on executive functioning, while oral language skills are mostly language proficiency tasks. Apparently, brain structures of preterm children are associated more strongly with language tasks than with executive functioning based language tasks.

\section{Influencing factors}

It is plausible that gestational age (GA) is an important influencing factor in the relation between brain and language, where lower GA leads to more atypical brain and language development and a relation between these two parameters would be more obvious. However, the populations of almost all included studies consisted of very preterm children with a gestational age of $<33$ weeks. One study [30] included a population of late preterm children only (34-36 weeks GA). The authors did not find a correlation with language, which is in accordance with the idea that higher GA leads to less atypical brain and language development. However, this 
lack of correlation might be a consequence of the fact that only the cerebellum was studied, which in many of the other studies was not correlated with language. Because of these considerations we cannot indicate an effect of GA from our study results.

Another factor that might have been of influence is the MRI methodology used. Overall, studies that used DTI as a MRI measurement reported more significant correlations than studies that used volume measurements only.

A third factor to take into account is sex. Several included studies presented results for boys and girls separately. We analyzed these results to search for similarities in boys and girls, but did not find consensus within these results. Therefore, we cannot draw a general conclusion about the role of sex in the correlation between brain and language.

\section{Strengths and limitations}

The strength of this review is that it provides a clear overview of the most comparable studies on the relation between language and brain structure in school-aged preterm children. This is a very complex subject because it covers two crossing parameters, which each in itself is complex and variable. We achieved to keep the most important factors relatively stable, such as age of the population, MRI scanner features, language tests used and population size. Also, our classification into three language domain groups resulted in a structured overview. We hope that this categorization will contribute to the validity of future correlational studies of brain function and language outcomes.

A possible limitation of the study is the risk of publication bias, i.e. studies may have analyzed more regions in the brain than reported in the result section. To partly adjust for this, when a certain region was mentioned in the methods section, but not addressed in the results section, we interpreted this as: no significant correlation found. And then, of course, performed studies that did not find any significant correlations may not have been published at all. Therefore, we highlighted the results presented by at least two study groups. When only one study looked into a certain relation-for example the relation between written language skills and the fasciculi-we did not highlight the outcome in our review results and discussion.

In our systematic review we chose to focus on the most commonly used MRI and DTI methods (structural T1- and T2-weighted sequences MRI and DTI). However, there are already some new models, such as non-tensor-based diffusion imaging analyses (e.g. high angular resolution diffusion imaging (HARDI)) that are very promising. HARDI is a new advanced model, which is an improvement with respect to DTI because it can deal with crossing fibers in voxels. It is successfully used very recently in a study with preterm children at term equivalent age [44]. The authors state that their findings suggest that differences in arcuate fasciculi micro-structure have a significant impact on language development and modulate the first stages of language learning. However, non-tensor-based diffusion imaging analyses were beyond the scope of our systematic review since the method is relatively new. Data are still limited and no studies are published in school-aged preterm children yet.

\section{Implications for further research}

For future research we would recommend to relate the brain on a microstructural level to oral language skills in preterm children. We would recommend to use language tests such as the CELF, since this test battery consists of a number of subsets that cover all oral language domains and can be subdivided in subcategories, such as perceptive and expressive language. We recommend to study these oral language subcategories separately and relate these and written language skills to brain structures. With respect to MRI/DTI measurements, longitudinal GM and WM analysis seems to be promising methods, highly relevant to longitudinal 
research in preterm children and the relation with their cognitive development. We also recommend to use non-tensor-based diffusion imaging analysis, since this new advanced model is an improvement with respect to DTI. Besides, we recommend to use principle component analysis (PCA) as an analytical approach, which might prevent random correlational findings and can actually lead to meaningful associations. PCA is a renowned method with a longstanding tradition which is now again increasingly and successfully being used in neonatal MRI studies to quantify the proportion of shared variance in the measured water diffusion parameters (MD, FA, $\lambda_{\text {ax }}$ and $\lambda_{\text {rad }}$ ) across the tracts [45]. Lastly, more consistent data collection and data sharing could lead to more and quality-assured knowledge in this research field.

\section{Conclusion}

This systematic review gives an overview of the extensive and diverse scientific literature on the associations between MRI brain measures and language outcome in children born preterm. Oral language skills and verbal fluency were shown to be associated with CC volume. Oral language skills are also associated with the UF. Overall, oral language skills are more obviously associated with several microstructural brain areas than are verbal fluency tasks, which are executive functioning based language tasks, and reading and spelling tasks. No associations were found between cerebellar volume and verbal fluency. The relation between oral language and written language with cerebellar development seems weak. The relation between preterm brain injury and language outcome could not be proven in studies that used brain damage scales. This most likely implies that not one single damaged brain area is responsible for atypical language development, but that several brain areas and their connections are essential. For future research we would recommend to study overall brain connectivity in combination with oral language skills, in which good quality management and data sharing will be crucial to enhance our shared knowledge and clinical opportunities.

\section{Supporting information}

S1 Text. Search protocol.

(DOCX)

S1 Table. Newcastle-Ottawa Quality Assessment Scale. (DOCX)

S2 Table. PRISMA 2009 checklist.

(DOC)

\section{Acknowledgments}

We thank Wichor Bramer for his help with the systematic search in the computerized databases.

\section{Author Contributions}

Conceptualization: Lottie W. Stipdonk, Marie-Christine J. P. Franken, Jeroen Dudink.

Formal analysis: Lottie W. Stipdonk, Jeroen Dudink.

Methodology: Lottie W. Stipdonk.

Writing - original draft: Lottie W. Stipdonk.

Writing - review \& editing: Lottie W. Stipdonk, Marie-Christine J. P. Franken, Jeroen Dudink. 


\section{References}

1. Goldenberg RL, Culhane JF, lams JD, Romero R. Epidemiology and causes of preterm birth. Lancet. 2008; 371(9606):75-84. https://doi.org/10.1016/S0140-6736(08)60074-4 PMID: 18177778

2. Saigal S, Doyle LW. An overview of mortality and sequelae of preterm birth from infancy to adulthood. Lancet. 2008; 371(9608):261-9. https://doi.org/10.1016/S0140-6736(08)60136-1 PMID: 18207020

3. Blencowe H, Lee AC, Cousens S, Bahalim A, Narwal R, Zhong N, et al. Preterm birth-associated neurodevelopmental impairment estimates at regional and global levels for 2010. Pediatr Res. 2013; 74 Suppl 1:17-34.

4. Aarnoudse-Moens CS, Weisglas-Kuperus N, van Goudoever JB, Oosterlaan J. Meta-analysis of neurobehavioral outcomes in very preterm and/or very low birth weight children. Pediatrics. 2009; 124 (2):717-28. https://doi.org/10.1542/peds.2008-2816 PMID: 19651588

5. Elgen SK, Leversen KT, Grundt JH, Hurum J, Sundby AB, Elgen IB, et al. Mental health at 5 years among children born extremely preterm: a national population-based study. Eur Child Adolesc Psychiatry. 2012; 21(10):583-9. https://doi.org/10.1007/s00787-012-0298-1 PMID: 22752364

6. van Noort-van der Spek IL, Franken M, Weisglas-Kuperus N. Language Functions in Preterm-Born Children: A Systematic Review and Meta-analysis. Pediatrics. 2012; 129(4):745-54. https://doi.org/10. 1542/peds.2011-1728 PMID: 22430458

7. Botting N, Powls A, Cooke RW, Marlow N. Attention deficit hyperactivity disorders and other psychiatric outcomes in very low birthweight children at 12 years. J Child Psychol Psychiatry. 1997; 38(8):931-41. PMID: 9413793

8. Elgen I, Sommerfelt K, Markestad T. Population based, controlled study of behavioural problems and psychiatric disorders in low birthweight children at 11 years of age. Arch Dis Child Fetal Neonatal Ed. 2002; 87(2):F128-32. https://doi.org/10.1136/fn.87.2.F128 PMID: 12193521

9. Somhovd MJ, Hansen BM, Brok J, Esbjorn BH, Greisen G. Anxiety in adolescents born preterm or with very low birthweight: a meta-analysis of case-control studies. Dev Med Child Neurol. 2012; 54(11):98894. https://doi.org/10.1111/j.1469-8749.2012.04407.x PMID: 22924489

10. Kwon SH, Scheinost D, Vohr B, Lacadie C, Schneider K, Dai F, et al. Functional magnetic resonance connectivity studies in infants born preterm: Suggestions of proximate and long-lasting changes in language organization. Dev Med Child Neurol. 2016; 58:28-34. https://doi.org/10.1111/dmcn.13043 PMID: 27027605

11. Kovachy VN, Adams JN, Tamaresis JS, Feldman HM. Reading abilities in school-aged preterm children: a review and meta-analysis. Dev Med Child Neurol. 2015; 57(5):410-9. https://doi.org/10.1111/ dmcn.12652 PMID: 25516105

12. de Kieviet JF, Zoetebier L, van Elburg RM, Vermeulen RJ, Oosterlaan J. Brain development of very preterm and very low-birthweight children in childhood and adolescence: a meta-analysis. Dev Med Child Neurol. 2012; 54(4):313-23. https://doi.org/10.1111/j.1469-8749.2011.04216.x PMID: 22283622

13. Farajdokht F, Sadigh-Eteghad S, Dehghani R, Mohaddes G, Abedi L, Bughchechi R, et al. Very low birth weight is associated with brain structure abnormalities and cognitive function impairments: A systematic review. Brain Cogn. 2017; 118:80-9. https://doi.org/10.1016/j.bandc.2017.07.006 PMID: 28802183

14. Feldman HM, Lee ES, Yeatman JD, Yeom KW. Language and reading skills in school-aged children and adolescents born preterm are associated with white matter properties on diffusion tensor imaging. Neuropsychologia. 2012; 50(14):3348-62. https://doi.org/10.1016/j.neuropsychologia.2012.10.014 PMID: 23088817

15. Travis KE, Ben-Shachar M, Myall NJ, Feldman HM. Variations in the neurobiology of reading in children and adolescents born full term and preterm. Neurolmage Clin. 2016; 11:555-65. https://doi.org/10. 1016/j.nicl.2016.04.003 PMID: 27158588

16. Gäddlin PO, Finnström $O$, Samuelsson S, Wadsby M, Wang C, Leijon I. Academic achievement, behavioural outcomes and MRI findings at 15 years of age in very low birthweight children. Acta Paediatr Int J Paediatr. 2008; 97(10):1426-32.

17. Yliherva A, Olsén $P$, Järvelin MR. Linguistic skills in relation to neurological findings at 8 years of age in children born preterm. Logop Phoniatr Vocology. 2001; 26(2):66-75.

18. Rushe TM, Rifkin L, Stewart AL, Townsend JP, Roth SC, Wyatt JS, et al. Neuropsychological outcome at adolescence of very preterm birth and its relation to brain structure. Dev Med Child Neurol. 2001; 43 (4):226-33. PMID: 11305398

19. Skranes JS, Vik T, Nilsen G, Smevik O, Andersson HW, Brubakk AM. Cerebral magnetic resonance imaging and mental and motor function of very low birth weight children at six years of age. NEUROPEDIATRICS. 1997; 28(3):149-54. https://doi.org/10.1055/s-2007-973692 PMID: 9266552 
20. Isaacs EB, Edmonds CJ, Chong WK, Lucas A, Morley R, Gadian DG. Brain morphometry and IQ measurements in preterm children. Brain. 2004; 127:2595-607. https://doi.org/10.1093/brain/awh300 PMID: 15371289

21. Nosarti C, Giouroukou E, Healy E, Rifkin L, Walshe M, Reichenberg A, et al. Grey and white matter distribution in very preterm adolescents mediates neurodevelopmental outcome. Brain. 2008; 131(1):205-17.

22. McCoy TE, Conrad AL, Richman LC, Brumbaugh JE, Magnotta VA, Bell EF, et al. The relationship between brain structure and cognition in transfused preterm children at school age. Dev Neuropsychol. 2014; 39(3):226-32. https://doi.org/10.1080/87565641.2013.874428 PMID: 24742312

23. Scott FE, Mechelli A, Allin MP, Walshe M, Rifkin L, Murray RM, et al. Very preterm adolescents show gender-dependent alteration of the structural brain correlates of spelling abilities. Neuropsychologia. 2011; 49(9):2685-93. https://doi.org/10.1016/j.neuropsychologia.2011.05.016 PMID: 21651922

24. Arhan E, Gucuyener K, Soysal S, Salvarli S, Gurses MA, Serdaroglu A, et al. Regional brain volume reduction and cognitive outcomes in preterm children at low risk at 9 years of age. Childs Nerv Syst. 2017; 33(8):1317-26. https://doi.org/10.1007/s00381-017-3421-2 PMID: 28484867

25. Parker J, Mitchell A, Kalpakidou A, Walshe M, Jung HY, Nosarti C, et al. Cerebellar growth and behavioural \& neuropsychological outcome in preterm adolescents. Brain. 2008; 131(5):1344-51.

26. Narberhaus A, Segarra D, Caldú X, Giménez M, Pueyo R, Botet F, et al. Corpus callosum and prefrontal functions in adolescents with history of very preterm birth. Neuropsychologia. 2008; 46(1):111-6. https://doi.org/10.1016/j.neuropsychologia.2007.08.004 PMID: 17897687

27. Nosarti C, Rushe TM, Woodruff PWR, Stewart AL, Rifkin L, Murray RM. Corpus callosum size and very preterm birth: Relationship to neuropsychological outcome. Brain. 2004; 127(9):2080-9.

28. Allin M, Matsumoto $\mathrm{H}$, Santhouse AM, Nosarti C, Alasady MHS, Stewart AL, et al. Cognitive and motor function and the size of the cerebellum in adolescents born very pre-term. Brain. 2001; 124(1):60-6.

29. Martinussen M, Flanders DW, Fischl B, Busa E. Segmental brain volumes and cognitive and perceptual correlates in 15-year-old adolescents with low birth weight. J Pediatr. 2009; 155(6):848-853. https://doi. org/10.1016/j.jpeds.2009.06.015 PMID: 19683725

30. Brumbaugh JE, Conrad AL, Lee JK, Devolder IJ, Zimmerman MB, Magnotta VA, et al. Altered brain function, structure, and developmental trajectory in children born late preterm. Pediatr Res. 2016; 80 (2):197-203. https://doi.org/10.1038/pr.2016.82 PMID: 27064239

31. Caldú $X$, Narberhaus $A$, Junqué $C$, Giménez $M$, Vendrell $P$, Bargalló $N$, et al. Corpus callosum size and neuropsychologic impairment in adolescents who were born preterm. J Child Neurol. 2006; 21(5):40610. https://doi.org/10.1177/08830738060210050801 PMID: 16901446

32. Northam GB, Liégeois F, Tournier JD, Croft LJ, Johns PN, Chong WK, et al. Interhemispheric temporal lobe connectivity predicts language impairment in adolescents born preterm. Brain. 2012; 135 (12):3781-98.

33. Mullen KM, Vohr BR, Katz KH, Schneider KC, Lacadie C, Hampson M, et al. Preterm birth results in alterations in neural connectivity at age 16 years. Neurolmage. 2011; 54(4):2563-70. https://doi.org/10. 1016/j.neuroimage.2010.11.019 PMID: 21073965

34. Andrews JS, Ben-Shachar M, Yeatman JD, Flom LL, Luna BEATRIZ, Feldman HM. Reading performance correlates with white-matter properties in preterm and term children. Dev Med Child Neurol. 2010; 52(6):e94-e100. https://doi.org/10.1111/j.1469-8749.2009.03456.x PMID: 19747208

35. Constable RT, Ment LR, Vohr BR, Kesler SR, Fulbright RK, Lacadie C, et al. Prematurely born children demonstrate white matter microstructural differences at 12 years of age, relative to term control subjects: An investigation of group and gender effects. Pediatrics. 2008; 121(2):306-16. https://doi.org/10. 1542/peds.2007-0414 PMID: 18245422

36. Kontis D, Catani M, Cuddy M, Walshe M, Nosarti C, Jones D, et al. Diffusion tensor MRI of the corpus callosum and cognitive function in adults born preterm. NeuroReport. 2009; 20(4):424-8. https://doi. org/10.1097/WNR.0b013e328325a8f9 PMID: 19218872

37. Skranes J, Vangberg TR, Kulseng S, Indredavik MS. Clinical findings and white matter abnormalities seen on diffusion tensor imaging in adolescents with very low birth weight. Brain. 2007.

38. Von Der Heide RJ, Skipper LM, Klobusicky E, Olson IR. Dissecting the uncinate fasciculus: disorders, controversies and a hypothesis. Brain. 2013; 136(Pt 6):1692-707. https://doi.org/10.1093/brain/awt094 PMID: 23649697

39. Beal DS, Lerch JP, Cameron B, Henderson R, Gracco VL, De Nil LF. The trajectory of gray matter development in Broca's area is abnormal in people who stutter. Front Hum Neurosci. 2015; 9:89. https://doi.org/10.3389/fnhum.2015.00089 PMID: 25784869

40. Sowell ER, Thompson PM, Leonard CM, Welcome SE, Kan E, Toga AW. Longitudinal mapping of cortical thickness and brain growth in normal children. J Neurosci. 2004; 24(38):8223-31. https://doi.org/10. 1523/JNEUROSCI.1798-04.2004 PMID: 15385605 
41. Squeglia LM, Jacobus J, Sorg SF, Jernigan TL, Tapert SF. Early adolescent cortical thinning is related to better neuropsychological performance. J Int Neuropsychol Soc. 2013; 19(9):962-70. https://doi.org/ 10.1017/S1355617713000878 PMID: 23947395

42. Wendelken C, O'Hare ED, Whitaker KJ, Ferrer E, Bunge SA. Increased functional selectivity over development in rostrolateral prefrontal cortex. J Neurosci. 2011; 31(47):17260-8. https://doi.org/10. 1523/JNEUROSCI.1193-10.2011 PMID: 22114292

43. Linkersdorfer J, Jurcoane A, Lindberg S, Kaiser J, Hasselhorn M, Fiebach CJ, et al. The association between gray matter volume and reading proficiency: a longitudinal study of beginning readers. $\mathrm{J}$ Cogn Neurosci. 2015; 27(2):308-18. https://doi.org/10.1162/jocn_a_00710 PMID: 25203270

44. Salvan P, Tournier JD, Batalle D, Falconer S, Chew A, Kennea N, et al. Language ability in preterm children is associated with arcuate fasciculi microstructure at term. Hum Brain Mapp. 2017; 38(8):3836-47. https://doi.org/10.1002/hbm.23632 PMID: 28470961

45. Telford EJ, Cox SR, Fletcher-Watson S, Anblagan D, Sparrow S, Pataky R, et al. A latent measure explains substantial variance in white matter microstructure across the newborn human brain. Brain Struct Funct. 2017; 222(9):4023-33. https://doi.org/10.1007/s00429-017-1455-6 PMID: 28589258 